\title{
147. Preliminary Note on the Chromosome Alterations by $X$-rays in Loxoblemmus arictulus, a Species of Grylloid Family.
}

\author{
By Kazuo Suzuki. \\ Anatomical Institute, Faculty of Agriculture, Tokyo Imperial University, \\ Komaba, Tokyo.
}

(Comm. by C. Ishikawa, M.I.A., Dec. 12, 1932.)

Recent experiments on the chromosomes with X-ray radiations have shown some striking alterations on the morphology of the same.

Thus Muller and Painter by their static experiments have shown that the morphology of chromosomes are greatly changed by the exposure of X-rays on the testis of Drosophila (Muller and Painter 1929). The results so far obtained by these investigators as well as by some others can be put under the following types; 1) Translocation, where a section of a chromosome becomes permanently attached to a nonhomologaus one, 2) duplication, in which one of the fragments becomes united with a homologous chromosome, 3) inversion of a portion of a chromosome, and 4) deletion of a chromosome fragment.

All these types of the displacement of the chromosomes are described by Muller and Painter in Drosophila, while the similar cases are recorded by Goodspeed (1930) in the chromosomes of Nicotiana. It thus shows that the effect of the X-radiation is similar both in animals and plants.

Similar treatment with $\mathrm{X}$-rays was carried out by the writer with Loxoblemmus arictulus, the result of which will be briefly described as follows. The reason of using this cricket is that the germ-cell has only seven pairs of chromosomes, each of which have a peculiar shape of its own, which enables us clearly to distinguish each other morphologically. The seven pairs of chromosomes are 4 J's, 2 V's, 6 rods, beside an $\mathrm{X}$.

The larvae used were those that have grown about four weeks after the hatching, and the conditions under which the experiments were carried out were as follows:-

Kilovolts ............................... 70

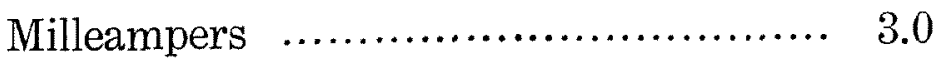

Distance from the taget............... $30 \mathrm{~cm}$.

Aluminum filter ........................ $1 \mathrm{~mm}$.

Time ................................. 15 minutes 
After the exposure to X-ray, the testis were taken out of the living insects and fixed with Flemming's strong solution or with Bouin's fluid.

The sections were cut with the thickness of 8-10 micra, and stained with Heidenhain's iron haematoxylin.

As with the experiments carried out by various investigators on animals and plants, a higher proportion of the alterations was observed when the larvae were killed some days after the treatment, mostly after three days.

Figure 1 represents a spermatogonia cell in which all the chromosomes are shown in their normal conditions. Figures 2-6 show the state of chromosomes induced by the treatment. Fig. 2, a b show the detachment of the short arms of $J$ chromosomes close to the attachment of the spindle fibers, the detached pieces are seen lying freely at the ends of the original chromosomes.

A large $\mathrm{Z}$ shaped chromosome represented by Fig. 3, a, has originated by the attachment of the longer arm of a $J$ chromosome at the end of the similar arm of another $J$, the detached piece is seen at the side of $\mathrm{Z}$ (Fig. 3, b). Such an attachment of a fragment of a chromosome to another one, leading to complete fusion of the same is never observed in normal and unaffected chromosomes.

A case where an $\mathrm{X}$-chromosome is divided into two nearly equal parts is seen in Fig. 3, 4, c, d, and in Fig. 4, a, a small piece is broken off from one end of an $\mathrm{x}$, while in Fig. 4, $\mathrm{b}$, a constriction is seen near one end of a chromosome which may represent a state of the detachment of the same as in a of the same figure, but it can also be considered as a case of a secondary union of a detached portion.

In Fig. 5, 6, the number of chromosomes has multiplied to from 15 to 17 instead of the normal number of 13 , some of which are rather small but quite distinct.

In Fig. 6, an irregular V-shapped chromosome a is seen which is apparently produced by fusion of three small detached pieces.

Thus it will be seen that all these variations in number as well as the morphology of the chromosomes are altered through the action of X-ray.

The present investigation is carried out under the direction of Prof. Dr. K. Masui, to whose kind guidance the author wishes to extend his most cordial thanks. 
No. 10.] Preliminary Note on the Chromosome Alterations by X-rays

\section{Literature.}

1929, Muller, H.J. and Painter, T.S.

The Cytological Expression of Changes in Gene Aligment produced by X-rays in Drosophila. Amer. Naturalist, Vol. 63.

1930, Goodspeed, T. H. and Avery, P.

Nature and Significance of Structural Chromosome Alterations Induced by $\mathrm{X}$-rays and Radium. Cytologia, I. 1931, Navashin, M.

A Preliminary Report on Some Chromosome Alteration by X-rays in Crepis. Amer. Naturalist, Vol. 65.

1931, Lewitzky, G.

Experimentally Induced Alteration of the Morphology of Chromosomes. Amer. Naturalist, Vol. 65.

\section{Explanation of plate.}

All the figures were drawn at the level of the desk on which the microscope was set with the aid of Abbes' drawing apparatus under magnification by using Leitz objective $1 / 12 \mathrm{~mm}$. and compensating ocular K. 13 , tube length $15 \mathrm{~mm}$.

Fig. 1. Normal spermatogonia with 12 chromosomes plus an $\mathrm{x}$.

Fig. 2-6. Spermatogonia treated with X-rays.

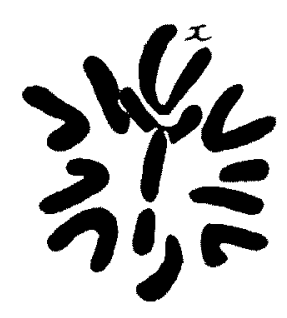

Fig. 1.

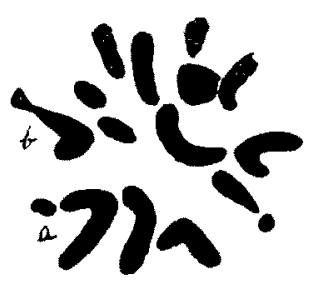

Fig. 4.

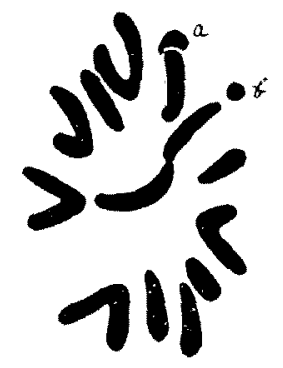

Fig. 2.

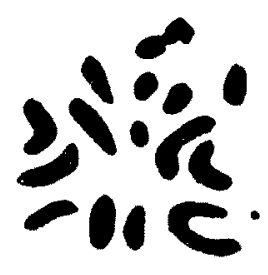

Fig. 5 .

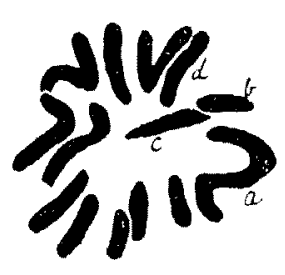

Fig. 3.

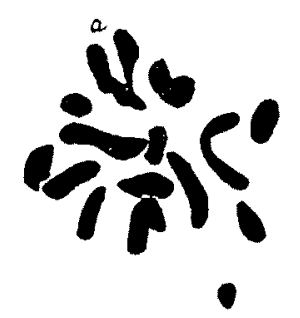

Fig. 6. 